\title{
Status of RENO-50
}

\section{Hyunkwan Seo ${ }^{1}$}

Seoul National University

1 Gwanak-ro, Gwanak-gu, Seoul 151-742, Korea

E-mail: hkseo16@gmail.com

RENO-50, 18 kton ultra-low-radioactivity liquid scintillator detector is proposed to determine the neutrino mass hierarchy and to make highly precise measurement of $\theta_{12}, \Delta \mathrm{m}_{21}{ }^{2}$ and $\Delta \mathrm{m}_{31}{ }^{2}$. The detector is located at roughly $50 \mathrm{~km}$ away from the Hanbit nuclear power plant in Korea where the neutrino oscillation due to $\theta_{12}$ takes place at maximum. The detector is expected to detect not only neutrinos from nuclear reactors but also neutrinos from the Sun, Supernova, the Earth, any possible stellar object and J-PARC neutrino beam. The experimental requirements and the strategy to achieve the main physics goals and sensitivities of RENO-50 are described.

XVI International Workshop on Neutrino Telescopes

2-6 March 2015

Palazzo Franchetti - Istituto Veneto, Venice, Italy

\section{${ }^{1}$ Speaker}




\section{Introduction}

An underground detector of RENO-50 under proposal will consist of 18,000 tons of ultralow-radioactivity liquid scintillator and 15,000 high quantum efficiency 20" photomultiplier tubes, located at roughly $50 \mathrm{~km}$ away from the Hanbit nuclear power plant in Korea where the neutrino oscillation due to $\theta_{12}$ takes place at maximum (see Figure 1). The detector is expected to detect neutrinos from nuclear reactors, the Sun, Supernova, the Earth, any possible stellar object and a J-PARC neutrino beam as well. It will be a multi-purpose and long-term operational detector, and also a neutrino telescope. The main goal is to determine the neutrino mass hierarchy and to measure the unprecedentedly accurate $(<1 \%)$ values of $\theta_{12}, \Delta \mathrm{m}_{21}{ }^{2}$, and $\Delta \mathrm{m}_{31}{ }^{2}$. It is also expected to detect $\sim 5,600$ events of a neutrino burst from a Supernova in our Galaxy, $\sim 1,000$ geo-neutrino events for 6 years, and 200 events of muon neutrinos from the J-PARC beam every year.

\section{R\&D for $3 \%$ energy resolution at $1 \mathrm{MeV}$}

The RENO-50 would be able to observe the manifestation of mass hierarchy in the oscillation effect if it establishes an extremely good energy resolution of $\sim 3 \%$ at $1 \mathrm{MeV}$. The energy resolution could be achieved based on maximized light yield of LS larger than 1,000 photoelectrons per $\mathrm{MeV}$, through making (a) an increased photosensitive area using 15,000 20" PMTs, (b) use of high (35\%) quantum efficiency PMTs, and (c) an increased attenuation length of LS up to $25 \mathrm{~m}$. Figure 2 shows the expected energy resolution with and without improvement in light yield, quantum efficiency and attenuation length.

\section{Study of precise $(<1 \%)$ determination of mixing parameters}

The high precision measurements of $\theta_{12}, \Delta \mathrm{m}_{21}{ }^{2}$, and $\Delta \mathrm{m}_{31}{ }^{2}$ can make a strong impact on explaining the pattern of neutrino mixing and its origin. It will also provide useful information on the effort of finding a flavor symmetry. Figure 3 shows the result of MC study for

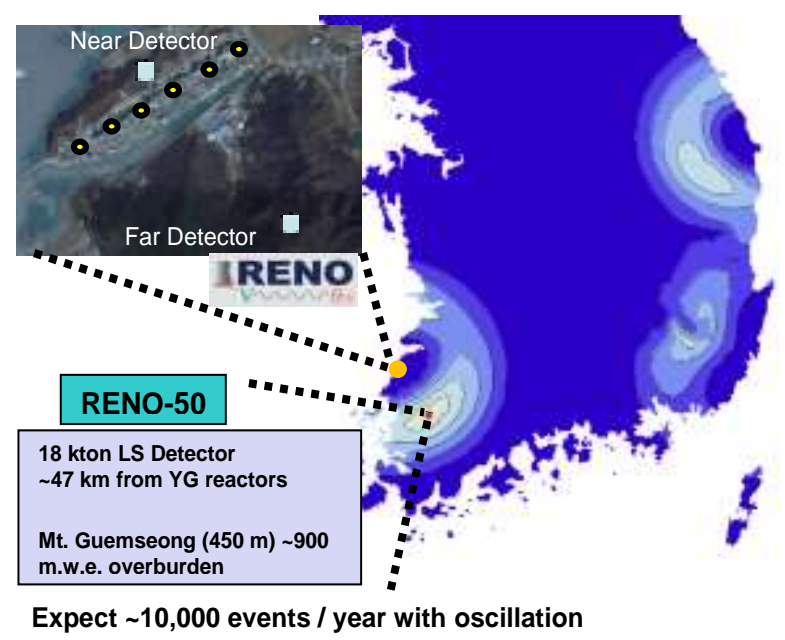

Figure 1. The RENO-50 detector will be located at underground of Mt. Geumseong in a city of Naju, $47 \mathrm{~km}$ from the Hanbit nuclear power plant. The contours of different colors indicate the sensitivity of mass hierarchy determination. 


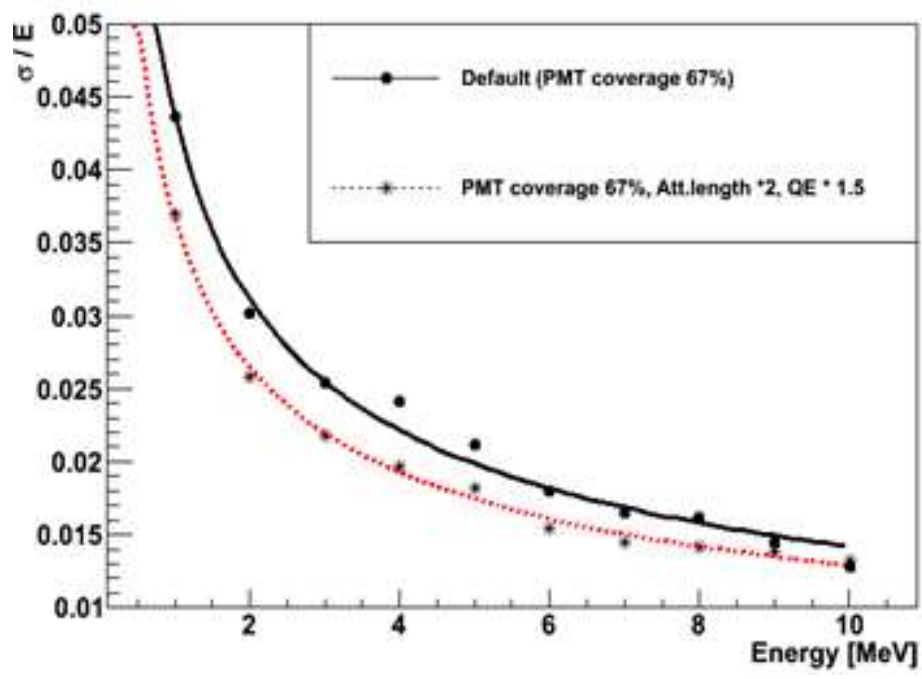

Figure 2. Expected energy resolution. Solid line is the case without improvement in light yield, quantum efficiency and attenuation length and dashed line is with improvement of them.

determination of mixing parameters. We expect to reach the following accuracies of measurements from 10 years data assuming fraction of background $=5 \%$, background error $=$ $8 \%$ and detection efficiency error $=1.0 \%$.

$$
\delta\left(\Delta \mathrm{m}^{2}{ }_{21}\right)=\sim 0.2 \%, \delta \sin ^{2}\left(2 \theta_{12}\right)=\sim 0.8 \%, \delta\left(\Delta \mathrm{m}^{2}{ }_{21}\right)=\sim 0.1 \%
$$

Figure 3 shows the sensitivity of the measurments vs. Year.

\section{Conclusion}

We have studied the requirements and the strategy to reach $3 \%$ energy resolution at 1 $\mathrm{MeV}$ and shown that high precision measurements $(<1 \%)$ of $\theta_{12}, \Delta \mathrm{m}_{21}{ }^{2}$, and $\Delta \mathrm{m}_{31}{ }^{2}$ are reachable by RENO-50. A R\&D funding (US \$2M) is given by the Samsung Science \& Technology Foundation from the end of 2014, and will continue in the next 3 years. R\&D efforts will be made on demonstrating feasibility of $3 \%$ energy resolution at $1 \mathrm{MeV}$, essential for determining the neutrino mass hierarchy. 
Status of RENO-50
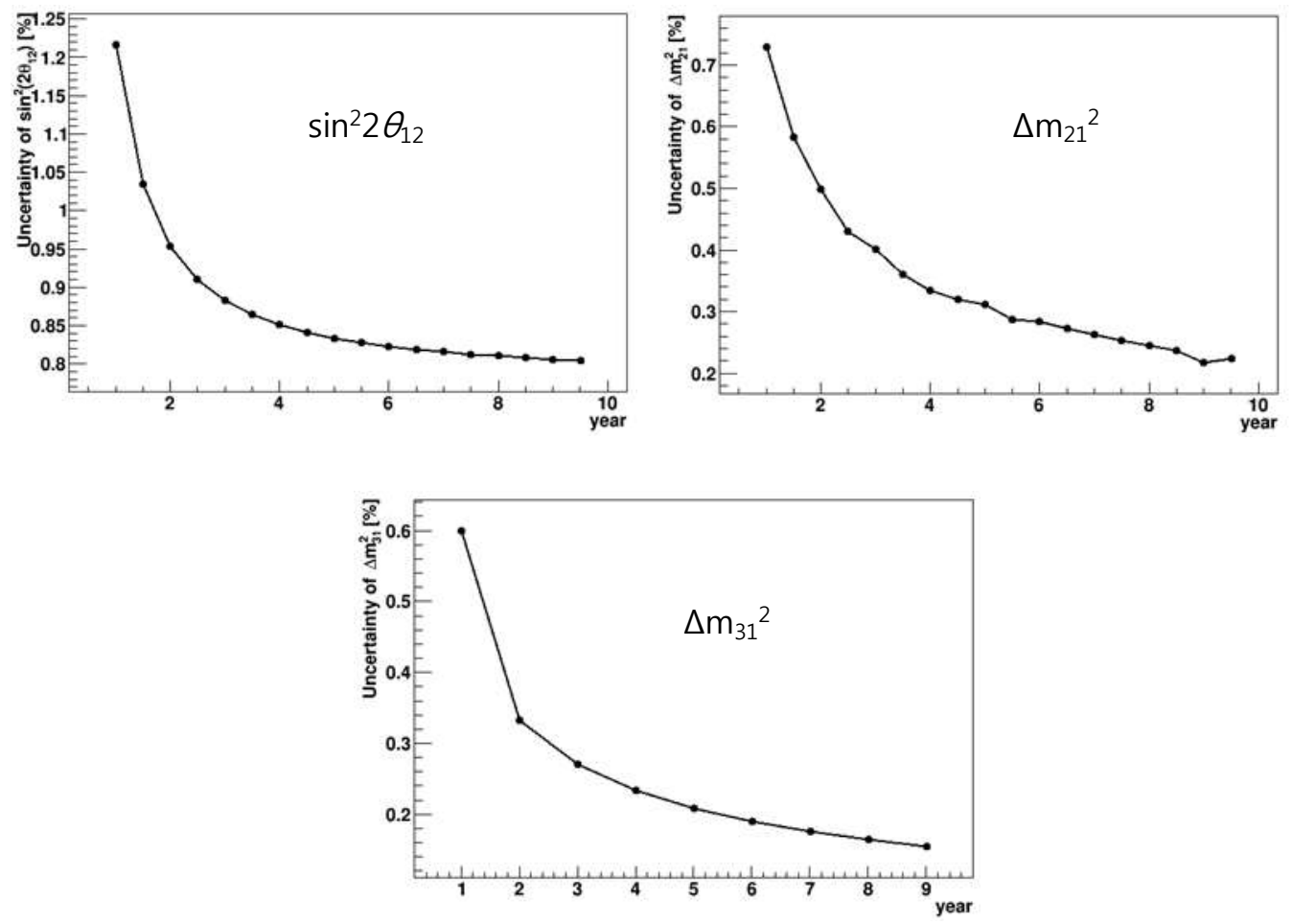

Figure 3. Sensitivity of the measurement of the mixing parameters vs. year. 